\title{
A Short Review of Gender Classification based on Fingerprint using Wavelet Transform
}

\author{
Sri Suwarno \\ Faculty of Information Technology \\ Duta Wacana Christian University \\ Yogyakarta, Indonesia
}

\author{
P. Insap Santosa \\ Dept. of Electrical Engineering and Information Technology \\ Gadjah Mada University, \\ Yogyakarta, Indonesia
}

\begin{abstract}
In some cases, knowing the gender of fingerprint owner found in criminal or disaster scene is advantageous. Theoretically, if the number of the male and female fingerprints in a database is equal, then the identification process of a fingerprint on that database would be two times faster. Some methods have been used to classify gender based on the fingerprint. Most of the method is based on ridge density. This method showed good result. However, it is sensitive to the location of the fingerprint area where ridge density is determined. This paper reviews some literature that used wavelet transform to generate features of a fingerprint. As far as what we found in literature, the number of papers on this topic is very limited. However, based on the literature we reviewed, wavelet transform gives some advantages compared to ridge density counting.
\end{abstract}

Keywords-Fingerprint; gender; ridge density; wavelet transform

\section{INTRODUCTION}

It is desirable to find features that can be used to determine people's gender. In general, it is believed that male and female have unique physical characteristics, such as fingerprint, palmprint, and face. Even in [1] and [2] writers reported that male and female shows the difference in the way they talk or walk. When gender can be detected based on their physical characteristics, unknown criminal or victims of natural disasters can be identified faster.

As one of the biometrics means, the fingerprint commonly classified into five major classes, namely whorl, left-loop, right-loop, arch and tented arch. It is advantageous when fingerprints further classified based on the gender of its owner. Furthermore, when the gender of the fingerprint is known then extracting and matching modules can be developed properly [3].

Many researchers have taken a close look to find fingerprint features that can be used to classify gender. They commonly determine ridge related features [4], [5], analyze frequency domain [6], and implement 2D Discrete Wavelet Transforms [7].

In this paper, we reviewed some literature that used wavelet transform to classify fingerprint based on its gender. Before that, we shortly reviewed some literature that used ridge features to do the same goal.

\section{GENDER CLASSIFICATION BASED ON RIDGE DENSITY}

Fingerprint has some features such as core, delta, ridge, and bifurcation, as seen in Fig. 1.

Core and delta, when existing, their relative position can be used as a feature. Ridges have more features such as thickness, orientation, and density. Meanwhile, bifurcations have orientation angles. These features are very helpful when the classification is done manually. However, when the classification is conducted manually, some preprocessing are needed.

Some researchers choose ridge density as a feature to classify gender. Acree [8] questioned if there is a gender difference in ridge density. To find the answer, he used 400 fingerprints picked randomly from 100 Caucasian males, 100 females, 100 African American males and 100 African American females within the age range of $18-67$. He reported that women tend to have higher ridge density than men. Furthermore, he wrote that a ridge density of 11 ridges $/ 25 \mathrm{~mm}$ or less tends to be a male, whereas fingerprint with a ridge density of 12 ridges $/ 25 \mathrm{~mm}$ or greater tends to be the female.

In India, Gungadin [9] observed a relationship between gender and ridge density by using 250 male and 250 female fingerprints taken in Karnataka, the southern part of India. He chooses the upper area of fingerprints within a square of $5 \mathrm{~mm}$ $\times 5 \mathrm{~mm}$ to determine the ridge density. He reported that fingerprint having less than 13 ridges $/ 25 \mathrm{~mm} 2$ more likely owned by a male, whereas a fingerprint having more than 14 ridges $/ 25 \mathrm{~mm} 2$ is more likely owned by a female.

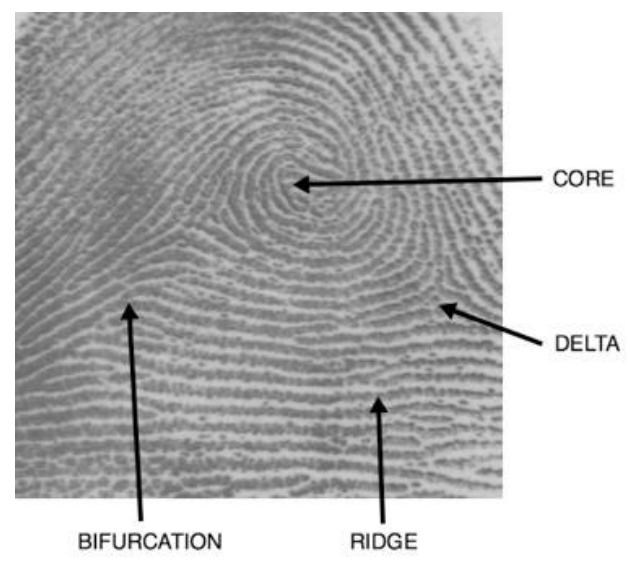

Fig. 1. Fingerprint features. 
He claimed that the result supported the hypothesis that male fingerprints tend to have lesser number of ridge compared to females.

In Spanish, Gutierrez-Romero, et al. [10] tried to prove that gender deferences based on fingerprint do exist. They collected all ten fingerprints of 100 males and 100 females of Spanish as a sample. To determine the ridge density, they used a square of $5 \mathrm{~mm} \times 5 \mathrm{~mm}$ area in three well-defined locations, namely radial, ulnar and lower. The result of their research demonstrated that significantly women tend to have higher ridge density than men.

The research of gender classification based on fingerprint was conducted in some countries, such as in sub-Saharan region [11], Turkey [12], [13], Thai [14], Malaysia [15] and much more.

All of the researchers mentioned above admitted that their findings are in a limited area. However, they believed that their finding is applied to another areas or countries. One of the advantages of this method is that classification can be done directly without using classification method.

Most of the methods chosen the specific location of the fingerprint and calculation of the ridge density calculated manually. Computer technology used only to enlarge fingerprint images and to calculate the result. When this method is applied in a computer program, needs much computation.

It is advantageous if all feature extraction and classification can be processed entirely using a computer. In this case, frequency domain analysis shows promising method. One of the methods is wavelet transform.

\section{GENDER CLASSIFICATION BASED ON WAVELET}

Wavelet can be used to extract features of the fingerprint. Extracting fingerprint features using wavelet decomposition gives some advantages. Today there are some wavelet transformations available. Furthermore, codes or even toolboxes available in major programming languages. These are some reasons why some researchers proposed the method to classify gender based on the fingerprint.

The role of transformation or decomposition is to generate some values that can be used to standardize the threshold. By using the threshold, classification can be done. The process of features generation using wavelet is as follows. Fingerprints are decomposed using a wavelet and resulting in some components. The number of components determined by the wavelet that used and the level of decomposition. Today there is some wavelet that can choose, such as Haar, Daubechies. The resulted components are used as a feature vector. By using the vector, the fingerprint can be classified using any classification methods.

Follows are some paper that reported the use of wavelet transformation to classify gender.

Shinde and Thepade [16] surveyed some gender classification based on a fingerprint that using wavelet decomposition. They analyze the gender of an individual by implementing Discrete Wavelet Transform (DWT) and
Singular Value Decomposition (SVD). The feature is generated by extracting the energy of all the sub-bands of DWT combined with the spatial features of non-zero singular values of the SVD of the fingerprint. They used 1000 fingerprints composed of 500 male and 500 female samples respectively. To clarify the gender, they implemented $\mathrm{K}$ nearest neighbor (KNN). The result of their work showed that the method gave accuracy up to $80.40 \%$ for male and $76.84 \%$ for female.

Kaur and Mazumbar [6] using Fast Fourier Transform (FFT), Discrete Cosine Transform (DCT), and Power Spectral Density (PSD) to generate fingerprint features. They argued that these transformations generate most of the information of the spatial domain image. This information can be used as features to estimate the gender by comparing them with predefined values. To prove the hypothesis they used 220 fingerprints composed of 110 female and 110 males. The predetermined values are set based on 50 chosen female and male fingerprints. The result of their research showed that the method gave $90 \%$ accuracy on 110 female samples and $79.09 \%$ on 110 male samples.

Gnanasivam and Muttan [17] implemented level 6 Discrete Wavelet Transform (DWT) and Singular Value Decomposition (SVD) to classify gender based on the fingerprint. They combined the energy of sub-band of DWT and the spatial features of non-zero singular values obtained from SVD as input to $\mathrm{K}$ nearest neighbor (KNN). They implemented the method to classify 3570 fingerprints composed of 1980 male and 1590 female fingerprints. All fingerprints are taken from all ten fingers. They used $2 / 3$ of them to train the system, and the rest was used in the classification stage. They reported that the method achieved an accuracy of $91.67 \%$ for male and $84.69 \%$ for female. Furthermore, they reported that little fingerprints showed higher success rate compared to other fingers.

Tom and Arulkumaran [7] used 2D- Discrete Wavelet Transform (DWT) combined with Principal Component Analysis (PCA) to generate a vector feature. By using this feature, they classify gender fingerprints by implementing the minimum distance method. They used fingerprints of 200 males and 200 females of various age groups. They reported that the success rate of the method is around $70 \%$.

\section{DISCUSSION AND CONCLUSION}

So far the common method to gender classification based on a fingerprint are use ridge density. They showed good result and used in some different countries. However, the methods need some preprocessing to enhance the clarity of the fingerprint. The enhancement is needed because most of the classification process was done manually. Although some researchers used automatic classification, however, the result was lower compared to manually classification.

Implementing wavelet transform to classify fingerprint based on its gender is promising. Some literature reported that wavelet transformation gives good result in generating features.

The methods can be implemented without using preprocessing. The methods work in the frequency domain, so the position or the orientation of the fingerprint are relatively 
irrelevant. The computation of wavelet decomposition is fast, and application or toolbox for that purpose is available.

\section{ACKNOWLEDGEMENT}

This paper is part of the research funded by The Ministry of Research, Technology and Higher Education of Indonesia.

REFERENCES

[1] K. Arai and R. A. Asmara, "Human Gait Gender Classification in Spatial and Temporal Reasoning," Int. J. Adv. Res. Artif. Intell., vol. 1, no. 6, pp. 1-6, 2012.

[2] K. Arai, "Human Gait Gender Classification using 3D Discrete Wavelet Transform Feature Extraction,” vol. 3, no. 2, pp. 12-17, 2014.

[3] M. Frick, S. K. Modi, S. J. Elliott, and E. P. Kukula, "Impact of gender on fingerprint recognition.," in 5th international conference on information technology and applications, 2008, no. Icita, pp. 717-721.

[4] V. C. Nayak, P. Rastogi, T. Kanchan, K. Yoganarasimha, G. P. Kumar, and R. G. Menezes, "Sex differences from fingerprint ridge density in Chinese and Malaysian population," Forensic Sci. Int., vol. 197, no. 1-3, pp. 67-69, 2010.

[5] E. OMidiora, O. Ojo, N. Yekini, and T. Tubi, "Analysis, Design and Implementation of Human Fingerprint Patterns System "Towards Age \& Gender Determination, Ridge Thickness To Valley Thickness Ratio (RTVTR) \& Ridge Count On Gender Detection," Int. J. Adv. Res. Artif. Intell., vol. 1, no. 2, pp. 57-63, 2012.

[6] R. Kaur and S. G. Mazumdar, "Fingerprint Based Gender Identification Using Frequency Domain Analysis," vol. 3, no. 1, pp. 295-299, 2012.

[7] R. J. Tom and T. Arulkumaran, "Fingerprint Based Gender Classification Using 2D Discrete Wavelet Transforms and Principal Component Analysis," Int. J. Eng. Trends Technol., vol. 4, no. 2, pp. 199-203, 2013.
[8] Mark A. Acree, "Is There a Gender Difference in fingerprint density ?," Forensic Sci. Int., vol. 102, pp. 35-44, 1999.

[9] S. Gungadin, "Sex Determination from Fingerprint Ridge Density," Internet J. Med. Updat., vol. 2, no. 2, pp. 4-7, 2007.

[10] E. Gutierrez-Redomero, C. Alonso, E. Romero, and V. Galera, "Variability of fingerprint ridge density in a sample of Spanish Caucasians and its application to sex determination," Forensic Sci. Int., vol. 180, no. 1, pp. 17-22, 2008.

[11] E. Guti??rrez-Redomero, J. A. Quir??s, N. Rivalder??a, and M. C. Alonso, "Topological Variability of Fingerprint Ridge Density in a SubSaharan Population Sample for Application in Personal Identification," J. Forensic Sci., vol. 58, no. 3, pp. 592-600, 2013.

[12] H. Oktem, A. Kurkcuoglu, I. C. Pelin, A. C. Yazici, G. Aktaş, and F. Altunay, "Sex differences in fingerprint ridge density in a Turkish young adult population: A sample of Baskent University," J. Forensic Leg. Med., vol. 32, pp. 34-38, 2015.

[13] E. B. Ceyhan and S. Sagiroglu, "Gender inference within Turkish population by using only fingerprint feature vectors," in 2014 IEEE Symposium on Computational Intelligence in Biometrics and Identity Management (CIBIM), 2014, pp. 146-150.

[14] P. Soanboon, S. Nanakorn, and W. Kutanan, "Determination of sex difference from fingerprint ridge density in northeastern Thai teenagers,” Egypt. J. Forensic Sci., vol. 6, no. 2, pp. 185-193, 2015.

[15] S. F. Abdullah, A. F. N. A. Rahman, and Z. A. Abas, "Classification of gender by using fingerprint ridge density in northern part of Malaysia," ARPN J. Eng. Appl. Sci., vol. 10, no. 22, pp. 10722-10726, 2015.

[16] M. K. Shinde and S. A. Annadate, "Analysis of Fingerprint Image for Gender Classification or Identification: Using Wavelet Transform and Singular Value Decomposition," 2015 Int. Conf. Comput. Commun. Control Autom., pp. 650-654, 2015.

[17] P. Gnanasivam and D. S. Muttan, "Fingerprint gender classification using wavelet transform and singular value decomposition," IJCSI Int. J. Comput. Sci. Issues, vol. 9, no. 2, 2012. 